\title{
To Perform an Axillary Lymph Node Dissection or Not? That Is (Still) the Question
}

\author{
Carla S. Fisher, MD, MBA, FACS \\ Department of Surgery, Indiana University School of Medicine, Indianapolis, IN
}

Management of the axilla is a topic very familiar, and perhaps frustrating, to surgeons treating breast cancer. Historically, the way we manage the axilla has been dictated by our view of the role it plays in breast cancer outcomes.

Dr. William Halstead popularized the idea that the axilla provides a "bridge" for the spread of cancer from the breast to the rest of the body. By intervening early with radical surgery, surgeons could effectively stop the spread of cancer. This view was challenged based on retrospective data that failed to show a survival advantage for aggressive nodal surgery. ${ }^{1}$

The randomized, prospective National Surgical Adjuvant Breast Project B-04 trial confirmed that the addition of axillary lymph node dissection (ALND) to mastectomy did not improve distant disease-free or overall survival (OS), but ALND continued because nodal status was important for making adjuvant chemotherapy decisions. The sentinel lymph node biopsy (SLNB) technique then allowed nodal status to be determined with less morbidity. Finally, the American College of Surgeons Oncology Group (ACOSOG) Z0011 trial highlighted the diminishing role of the axilla in treatment decision-making. Therefore, in the modern era, what is the role of the axilla in the treatment of breast cancer?

This issue of Annals of Surgical Oncology reports the findings of a team from Memorial Sloan Kettering Cancer Center (MSKCC) that retrospectively analyzed T1-T2cN0 breast cancer patients undergoing SLNB and ALND. ${ }^{2}$ In a

(C) Society of Surgical Oncology 2020

First Received: 19 May 2020;

Published Online: 4 June 2020

C. S. Fisher, MD, MBA, FACS

e-mail: fishercs@iu.edu study of patients between 2010 and 2018, Mamtani et al. identified a specific pathologic finding that significantly predicted an increased risk for patients with four or more positive non-sentinel lymph nodes (NSLNs). Among 1114 patients who underwent both SLNB and ALND, $113(10 \%)$ were noted to have extracellular tumor deposits (ETDs), defined as intravascular tumor emboli or metastatic deposits in the axillary fat. Of these patients, 925 met the Z0011 criteria, specifically with one or two positive SLNs, and $122(13 \%)$ of these patients ultimately had four or more NSLNs. Based on the strong and significant association of ETDs with four or more positive NSLNs, the authors concluded that the presence of ETDs indicates a need or consideration for ALND. What this study did not demonstrate is whether removal and identification of additional NSLNs is necessary for improving patient outcomes and/or choosing adjuvant therapy.

A study of 9521 patients using the Surveillance, Epidemiology, and End Results (SEER) database showed no difference in breast cancer-specific survival or OS between ALND and SLNB used to treat T1-T2 breast cancer patients with three or more metastatic lymph nodes. ${ }^{3}$ Based on this, they concluded that ALND is limited to a staging procedure for this group of patients.

The group from MSKCC also published data indicating another pathologic feature, microscopic extracellular extension (mECE), found in lymph nodes associated with four or more additional positive NSLNs. ${ }^{4}$ Once again, they concluded that mECE may indicate consideration for ALND or axillary radiation. Importantly, they recently published clinical outcomes for patients with $\mathrm{mECE}$ showing low nodal recurrence rates with SLNB performed alone, even in the absence of radiation..$^{5}$ They recommend that the presence of $\mathrm{mECE}$ should not be a routine indication for ALND. 
Similar to the Mamtani et al. patients with one or two positive SLNs, in the Z011 trial, $13.7 \%$ of the patients who underwent ALND had four or more NSLNs. ${ }^{6}$ This indicates that a similar number of patients who did not undergo ALND had a similar nodal burden, and the equivalent outcomes for both groups of patients is well-known. ${ }^{7}$

Although we do not know the clinical outcomes for the patients in this current analysis, we can surmise a significant rate of lymphedema for this group of patients. If the National Comprehensive Cancer Network (NCCN) guidelines had been followed, the patients with four or more positive lymph nodes also would have undergone axillary radiation. ${ }^{8}$ With both axillary interventions, lymphedema rates of $33.4 \%$ have been reported. ${ }^{9}$ This suggests surgical morbidity without a proven oncologic benefit.

In the closing comments of the published Z0011 trial, a landmark trial of the way we think about the role and subsequent management of the axilla for breast cancer patients, the authors state the following: "The only additional information gained from ALND is the number of nodes containing metastases. This prognostic information is unlikely to change systemic therapy decisions and is obtained at the cost of a significant increase in morbidity. The only rationale for ALND in these patients would be if the finding of additional nodal metastases would result in changes in systemic therapy."6 Finding both ETDs and mECE at the time of SLNB suggests a higher burden of disease in the axilla than the absence of either of these findings. They can provide an additional point of information that surgeons, medical oncologists, and radiation oncologists can use when making decisions about the multidisciplinary treatment of the patient. Nothing has been found to suggest that an ALND improves outcomes for patients with ETDs. Therefore, it may be considered but should not be mandated.

\section{REFERENCES}

1. Devitt JE. The influence of conservative and radical surgery on the survival of patients with breast cancer. Can Med Assoc J. 1962;87:906-10.

2. Mamtani A, Barrio AV, Goldman DA, Wen HY, Vincent A, Morrow M. Extranodal tumor deposits in the axillary fat indicate the need for axillary dissection among T1-T2cN0 patients with positive sentinel nodes. Ann Surg Oncol. 2020. https://doi.org/10. 1245/s10434-020-08632-1.

3. Bonneau C, Hequet D, Estevez JP, Pouget N, Rouzier R. Impact of axillary dissection in women with invasive breast cancer who do not fit the Z0011 ACOSOG trial because of three or more metastatic sentinel lymph nodes. Eur $J$ Surg Oncol. 2015;41:998-1004.

4. Gooch J, King TA, Eaton A, et al. The extent of extracapsular extension may influence the need for axillary lymph node dissection in patients with T1-T2 breast cancer. Ann Surg Oncol. 2014;21:2897-903.

5. Barrio AV, Downs-Canner S, Edelweiss M, et al. Microscopic extracapsular extension in sentinel lymph nodes does not mandate axillary dissection in Z0011-eligible patients. Ann Surg Oncol. 2020;27:1617-24.

6. Giuliano AE, Hunt KK, Ballman KV, et al. Axillary dissection vs no axillary dissection in women with invasive breast cancer and sentinel node metastasis: a randomized clinical trial. JAMA. 2011;305:569-75.

7. Giuliano AE, Ballman KV, McCall L, et al. Effect of axillary dissection vs no axillary dissection on 10-year overall survival among women with invasive breast cancer and sentinel node metastasis: the ACOSOG Z0011 (Alliance) randomized clinical trial. JAMA. 2017;318:918-26.

8. National Comprehensive Cancer Network. 2020. https://www.ncc n.org/professionals/physician_gls/pdf/breast.pdf. Accessed 12 May 2020.

9. Johnson AR, Kimball S, Epstein S, et al. Lymphedema incidence after axillary lymph node dissection: quantifying the impact of radiation and the lymphatic microsurgical preventive healing approach. Ann Plast Surg. 2019;82(4S Suppl 3):S234-41.

Publisher's Note Springer Nature remains neutral with regard to jurisdictional claims in published maps and institutional affiliations.

DISCLOSURE There are no conflicts of interest. 